IRA-International Journal of Management \& Social Sciences

ISSN 2455-2267; Vol.06, Issue 03 (2017)

Pg. no. 411-418

Institute of Research Advances

https://research-advances.org/index.php/RAJMSS

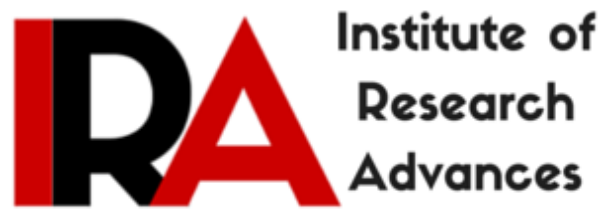

\title{
Management of Change Delivery Decision Making Through the Force Field Analysis in Georgia
}

\author{
Jilda Cheishvili ${ }^{1}$, Irene Zarnadze ${ }^{2}$, Shalva Zarnadze ${ }^{3}$ \\ $\mathbf{1 , 2 , 3}$ Department of Public Health, Healthcare Management, Policy and Economy \\ Tbilisi State Medical University, Georgia.
}

Type of Review: Peer Reviewed.

DOI: http://dx.doi.org/10.21013/jmss.v6.n3.p7

\section{How to cite this paper:}

Cheishvili, J., Zarnadze, I., \& Zarnadze, S. (2017). Management of Change Delivery Decision Making Through the Force Field Analysis in Georgia. IRA-International Journal of Management \& Social Sciences (ISSN 2455-2267), 6(3), 411-418. doi:http://dx.doi.org/10.21013/jmss.v6.n3.p7

(C) Institute of Research Advances

(c) BY-NC

This work is licensed under a Creative Commons Attribution-Non Commercial 4.0 International License subject to proper citation to the publication source of the work.

Disclaimer: The scholarly papers as reviewed and published by the Institute of Research Advances (IRA) are the views and opinions of their respective authors and are not the views or opinions of the IRA. The IRA disclaims of any harm or loss caused due to the published content to any party. 


\begin{tabular}{l} 
ABSTRACT \\
$\begin{array}{l}\text { Children's generation of Georgia recovers parent's generations only for 70-80\%. According to same } \\
\text { data, there are only } 14 \text { countries with the similar low indicators. The increase of C-section can cause } \\
\text { the decrease of birthdates. } \\
\text { WHO recommended The Partnership for Maternal, Newborn, and Child Health-through the creation } \\
\text { of an Advocacy Team. (WHO, 2005). This model ensures the relaxed environment where the mothers } \\
\text { can receive information delivered by qualified health workers, who teach pregnant women and family } \\
\text { members about health and well-being. Advocacy Team comprises - nurses, medical students, and } \\
\text { volunteer. } \\
\text { Project benefit will be increased antenatal and postnatal care services through the country, in the } \\
\text { lead the better attitude and knowledge about health and well-being across all Social levels. Further } \\
\text { families will save money because they couldn't pay for C-section } \\
\text { Evaluation of the project -annual statistical analysis by NCDC (National Center for Disease Control } \\
\text { and Public Health) decrease C-section rate for mother request. } \\
\text { Project outcome will be increased maternal and Child health and well-being, Increase right } \\
\text { participation from the community, Support of pregnant women and her family economically. }\end{array}$ \\
\hline
\end{tabular}

\section{Background}

Managing change is seen as being skilled at creating, acquiring and transferring knowledge thereby modifying its behavior to reflect new knowledge and insights (Garvin 1993). ${ }^{(1)}$

Pregnant women receive the services that are progressively changing. Traditional these services have an ever-increasing management responsibility, and it is important when considering change management to reflect upon potential benefits not only for the pregnant women but also for population and the organization as a whole.

The theory of change management draws on a number of social science disciplines and tradition. This makes defining core concepts difficult, therefore, several models/ theories were considered for guidance when planning a proposal for a change. ${ }^{(2)}$

\section{Actuality of the topic}

According to Statistical analysis in Georgia population level continues to reduce dramatically and for 2015 it is 3, 729,5. (Geostat,2015). (Figure N 1). According to the UNICEF Population in Georgia will drop to $28 \%$ by 2050 . 

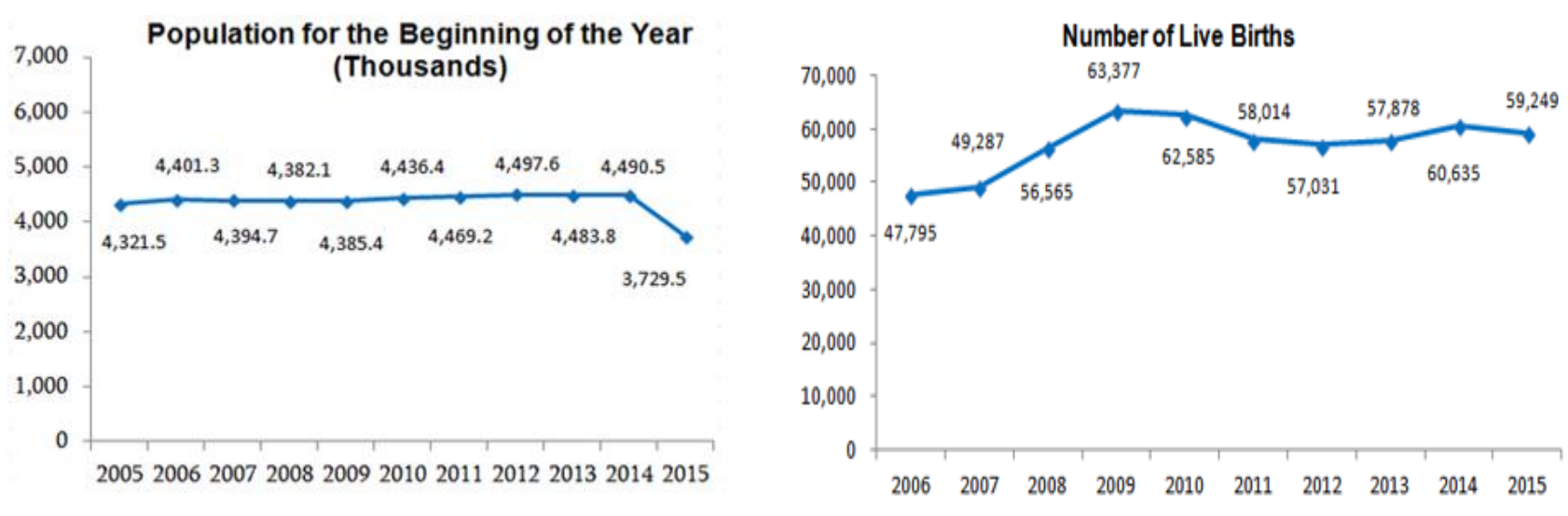

Figure N1

Figure N2

Children's generation of Georgia recovers parent's generations only for 70-80\%. According to same data, there are only 14 countries with the similar low indicators. The increase of $\mathrm{C}$-section can cause decrease of birthdates.(Figure N2 ).

According to the National Centre for Disease control and Public health in 2014 deliveries were registered $57 \%$ were physiological and $43 \%$ - pathological.

In 2014 were performed 23,369 caesarean sections in Georgia during the last decade, the

Increase of the number of caesarean sections was observed in Georgia, like in the majority of

Developed countries. (Figure N3,N4).

C- Section is one of the most commonly performed surgical operations in the world. Obstetricians and scientists view C-section as a high-risk intervention. Approximately $40 \%$ of C-sections are because of the mother's request (without any medical indication) in Georgia.

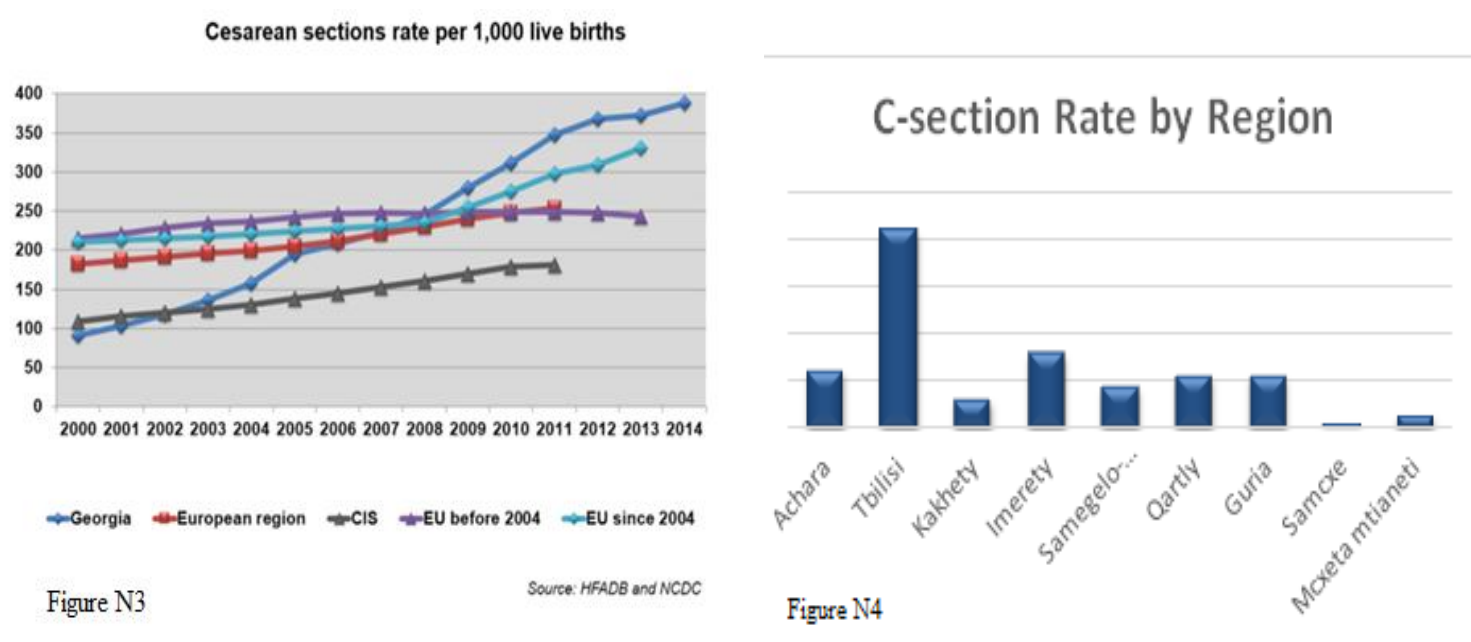

Assessment of the quality of Perinatal Care was conducted in order to promote effective perinatal care practices. From 2015, a 50 regionalization of perinatal services (introduction of levels of care), supported by the USAID/Sustain, began in Imereti and Racha-Lechkhumi. In Georgia in some private clinics number of C-sections is up to 70-80\%. 
The World Health Organisation recommendation is C-sections should not exceed 10-15\%. CSection rates higher than $10 \%$ are associated with long-term maternal and prenatal morbidity. (WHO, 1985).

In 2012 National Centre for Disease control and Public health (NCDC) have done the research about main reasons of choosing $\mathrm{C}$-section in pregnant women and her family members in Georgia and the result is they are choosing $\mathrm{C}$-section to reduce the pain during labour.

"In the last decades, the proportion of birth by caesarean section has increased in an unprecedented way. This is a multifactorial phenomenon and in many cases country- and culture-specific. Caesarean section has become a very safe procedure in many parts of the world to the point of considering it almost infallible. Some of the most omnipresent reasons behind this rise are the fear of pain during birth including the pain of uterine contractions, the convenience to schedule the birth when it is most suitable for families or health care professionals, or because it is perceived as being less traumatic for the baby. In some cultures, caesarean section allows choosing and setting the day of the birth according to certain believes of luck or better auspicious for the newborn's future. In many countries, societal consensus has imposed a demand for the perfect outcome and doctors are sued when the results are not as expected fueling the fear of litigation. In addition, in some societies, delivery by caesarean section is perceived to preserve better the pelvic floor resulting in less urinary incontinence in the future or sooner and more satisfactory return to sexual life." (WHO,2015)

More than fifty years obstetrician science gives recommendation for pregnant women use epidural anesthesia for reducing pain during labour. ${ }^{(3)}$

There is no evidence and any research why women did not choose epidural anesthesia, and why they prefer C-section for reducing pain during labour. Through the internet I have done the research to define the core of difficulties knowledge about epidural anesthesia trough the population.

From 220 respondent: most of them have been delivered (78\%),all of them knows that epidural anesthesia is for reducing pain during labour, $61 \%$ from respondent think that it has not any complication, $5 \%$ did not understood what is epidural anesthesia (when medical staff explained it) and $62 \%$ think that epidural anesthesia causes reduce the pain just only second period of delivery, a first period of delivery (which is hold 14-16 hours) is the same painful as in case of withought epidural labour.

This research is not valid because it has done just only though the internet, but we can see the main picture. Pregnant women have not enough knowledge about types of anesthesia, complications and worse outcomes in each cases of delivery type.

\section{The Aim of the Research}

To increase the knowledge and attitude during the pregnant period World Health Organisation (WHO) gives recommendation to establish the Partnership for Maternal, Newborn and Child Health - though the creation of an Advocacy Team. (WHO, 2005).

The vision (Value, Innovation, safety, Involvement, Observation, Noticeable) of the Advocacy Team should be that an every woman and newborn in every setting area should be realise their rights to physical and mental health and wellbeing, social and economic opportunities and should able to participate fully in shaping prosperous and sustainable societies.

The aim of Advocacy Team should be to increase the engagement, alignment and accountability of pregnant women and her family, by creating a multi-stakeholder platform that will support the successful implementation of the Global Strategy for Women's, and Children's Health, enabling partners to achieve more together than any individual partner could do alone. 
The Advocacy Team will be focus on pregnant women/ her family members, and community, they will: Increase knowledge of pregnant women and her family members to make right decision about delivery type.

The Partnership will fully commit to all of the 2030 targets in the Global Strategy. ${ }^{(4)}$ During the life of this Strategic Plan, the focus will be on driving progress on the targets of direct relevance to the continuum of care across maternal and newborn health:

- $\quad$ Reduce global maternal mortality [SDG3.1]

- $\quad$ Reduce child mortality [SDG3.2]

- Reduce newborn and infant mortality [SDG3.2]

To achieve this progress, the Partnership will necessarily strengthen inter-sectoral outreach and collaboration on other Global Strategy and SDG targets. ${ }^{(5)}$

This model ensures the relaxed environment where the mothers can receive information delivered by qualified health workers, who teach pregnant women and family members about health and wellbeing.

Advocacy Team should be comprises: nurses, medical students and volunteer.

\section{Literature Review}

Force field Analysis - Kurt Lewin's Model

Sociologist Kurt Lewin developed a 'force field analysis' model (1951) which describes any current level of performance or being as a state of equilibrium between the driving forces that encourage upward movement and the restraining forces that discourage it. Essentially this means that a current equilibrium exists because the forces acting for change are balanced by the forces acting against change. ${ }^{(6)}$

- The driving forces are (usually) positive, reasonable, logical, conscious and economic.

- The restraining forces are (usually) negative, emotional, illogical, unconscious and social/psychological.

Both sets of forces are very real and need to be taken into account when dealing with change, or managing change, or reacting to change. (Figure N5)

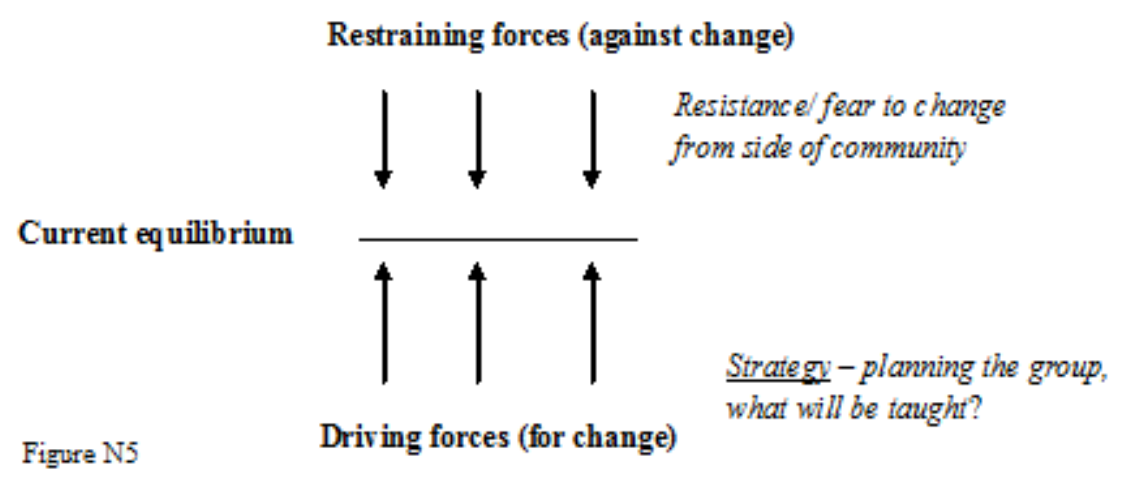


This theory places emphasis on the driving and resisting forces associated with any change, and to achieve success the importance lies with ensuring that driving forces outweigh resisting forces. Driving forces tend to initiate change or keep it going whereas restraining forces act to restrain or decrease the driving forces. ${ }^{(7)}$

Lewin suggested that modifying the forces which maintain the status may be easier than increasing the forces for change ${ }^{(8)}$

- Actual: To create of Advocacy Team

- Optimal: Controlled Advocacy Team allocation promoting continuity.

- Problem: Negative predispose from community

- Goal: Better attitude and knowledge about health and wellbeing across all Social level

\section{Lewin's change model}

Lewin's model has 3 steps: unfreezing - reducing strength of forces which maintain current equilibrium, moving - developing new organizational values, attitudes and behaviors to help move the organization on, and refreezing - stabilizing after the changes have been made so that there's a new equilibrium. ${ }^{(9)}$

If we are going to create Advocacy Team for implementation new service for community we can review Lewin's change model for each of steps: ${ }^{(10)}$

1. Unfreeze

To create the right environment for change

$\checkmark$ Assess current practice and service users from Advocacy Team.

$\checkmark$ Evidence based materials to be provided ensure success.

2. Change

\section{Create the intended change}

$\checkmark$ Introduce the new project to one of the maternal delivery houses, and pregnant women who are going to deliver there.

\section{$\checkmark$ Gain feedback from service users and staff}

3. Refreeze

\section{Reinforce and anchor the change}

$\checkmark$ Implement the programs to run and have teaching package in place.

$\checkmark$ Continuous evaluation to ensure the change is a positive one with the service users.

$\checkmark$ Regular evaluation with the staff about the change.

The first step of the project is to create team , because this project should be implement in a pilot region -small team should be enough and all team members vision for project: Value, Innovation, Safety, Noticeable, involvement, observation should be the same. ${ }^{(11)}$ 


\section{ACTION CENTERED LEADERSHIP Adair (1973)}

\begin{tabular}{|l|l|l|}
\hline Task & $\underline{\text { Team }}$ & $\underline{\text { Individual }}$ \\
To define the task. & Build team spirit. & \\
Form a plan. & Encourage \& motivate the team. \\
Allocate work \& Resources. & Appoint sub leader/s. & Develop individuals. \\
Control quality of the work. & Ensure good communication. & Praise individuals. \\
Motivate staff. & Develop the group. & Attend to personal problems. \\
\hline
\end{tabular}

Participative change, although a slow process is intended to last, if women believe in what they are doing, so creating an intrinsically imposed change. In order to achieve these four steps of the cycle were utilized. ${ }^{(12)}$

These being:

-Pregnant women were initially provided with the knowledge;

- Intent that they would develop a positive attitude on the proposal;

- Once individuals accept and are willing to try then they will influence the rest of the community;

Findings

In order to evaluate effectively there was the need to look at the change in terms of:

- Effect on the individual;

- Effect on the Team;

- Effect on the Maternity House.

Evaluation will be define by statistical analysis- for the C-section rate, from the hospital where this intervention will involved and by annual statistical analysis by decrease $\mathrm{C}$-section rate.

\section{Result and Outcomes:}

Result of this project should be: Increasing number of antenatal and postnatal care services thorough the country, in lead the better attitude and knowledge about health and wellbeing across all Social levels. Further families will save money, because they couldn't pay for C-section and formula feeding as a result of failure of breastfeeding.

The outcome will be: Increase maternal and child health and wellbeing, Increase right participation from the community, Support of pregnant women and her family economically. 


\section{References}

1. Sanchez-Sesma, F. J., Iturraran-Viveros, U., \& Kausel, E. (2013). Garvin's generalized problem revisited. Soil Dynamics and Earthquake Engineering, 47, 4. doi:10.1016/j.soildyn.2012.11.006

2. Hayes, J. (2014). The theory and practice of change management (4th ed.). Basingstoke: Palgrave Macmillan

3. Pundir, J., \& Coomarasamy, A. (2016). Obstetrics: Evidence-based algorithms. Cambridge: Cambridge University Press

4. Griggs.D (2013),Sustainable Development Goals for people and planet>nature 495(7441395)

5. Madeley, J. (2015). Sustainable development goals. Appropriate Technology, 42(4), 32

6. Burnes, B., \& Cooke, B. (2013). Kurt lewin's field theory: A review and Re- evaluation.International Journal of Management Reviews, 15(4), 408-425. doi:10.1111/j.1468-2370.2012.00348

7. Bond, M. H. (2013). Refining lewin's formula: A general model for explaining situational influence on individual social behavior. Asian Journal of Social Psychology, 16(1), 1-15. doi:10.1111/ajsp.12012

8. Eastman, C. A. (2012). Working with toshiba, lewin and dewey: A journey into the heart of change.Higher Education, Skills and Work-Based Learning, 2(2), 132-140. doi:10.1108/20423891211224612

9. Manchester, J., Miceli, D. L. G., Metcalf, J. A., Paolini, C. A., Napier, A. H., Coogle, C. L., \& Owens, M. G. (2014). Facilitating lewin's change model with collaborative evaluation in promoting evidence based practices of health professionals. Evaluation and Program Planning, 47, 82. doi:10.1016/j.evalprogplan.2014.08.007

10. Cummings, S., Bridgman, T., \& Brown, K. G. (2016). Unfreezing change as three steps: Rethinking kurt lewin's legacy for change management. Human Relations, 69(1),33

11. Jones, R. (2015). Quantitative measurement of blood loss during delivery. Journal of Obstetric, Gynecologic, \& Neonatal Nursing, 44(s1), S41-S41. doi:10.1111/1552-6909.12597

12. Braun, F. C., Avital, M., \& Martz, B. (2012). Action-centered team leadership influences more than performance. Team Performance Management: An International Journal, 18(3/4), 176-195. doi:10.1108/13527591211241015 